\title{
Fatal Pneumonitis Induced by Oxaliplatin: Description of Three Cases
}

\author{
L.B. Pontes D.P.D. Armentano A. Soares R.C. Gansl \\ Department of Medical Oncology, Albert Einstein Israelit Hospital, São Paulo, \\ Brazil
}

\section{Key Words}

Interstitial pneumonia $\cdot$ FOLFOX7m · Oxaliplatin · Drug-induced toxicities

\begin{abstract}
We describe 3 fatal cases of interstitial pneumonitis rapidly evolving to pulmonary fibrosis and death after the administration of oxaliplatin as part of the FOLFOX regimen. Due to the widespread use of oxaliplatin in oncology, clinicians should be aware of the risk and severity of oxalipatin-induced interstitial pneumonia.
\end{abstract}

\section{Introduction}

Lung toxicity associated with anticancer drugs is common and may include a variety of clinical syndromes. Oxaliplatin is a third-generation platin derivative which has changed clinical practice in the treatment of colorectal cancer; it is used in combination with 5 fluorouracil (5-FU) in the so-called FOLFOX regimen [1].

Clinical and preclinical studies established that its toxicity profile consists mainly of peripheral sensory neuropathy, hematological and gastrointestinal side effects [2]. These studies on safety found no significant increase in pulmonary complications. Recently, however, reports have been published about acute interstitial pulmonary disease and pulmonary fibrosis associated with oxaliplatin infusion [3].

Herein, 3 additional cases of FOLFOX-induced interstitial pneumonia are reported.

\section{Case Report}

Case 1

A 73-year-old female patient presented with consumptive syndrome and anemia. She had a positive history of cigarette smoking lasting almost 40 years, having discontinued the habit during the 
last 2 years. A colonoscopy/biopsy showed a transverse colon adenocarcinoma, and subsequent staging with CT scans revealed the presence of multiple hepatic nodules consistent with metastatic disease. There was no neoplastic lung involvement.

She was then treated with FOLFOX7m (oxaliplatin $85 \mathrm{mg} / \mathrm{m}^{2}$ on day $1+$ folinic acid $200 \mathrm{mg} / \mathrm{m}^{2} \mathrm{on}$ day $1+5$-FU 2,400 mg/m² over 46 h every 2 weeks) in combination with bevacizumab, with the first cycle in November 2007. The patient initially achieved partial response, with shrinkage of hepatic nodules, and then stable disease. After the tenth cycle, her disease remained stable and, because of grade II sensory neuropathy, oxaliplatin was stopped and the treatment continued with the 5-FUbased schedule plus bevacizumab. In March 2009, progressive hepatic disease was detected by routine assessment. Oxaliplatin was again added to the chemotherapeutic regimen, just as described above.

In December 2009, after the eleventh cycle was administered, the patient was admitted due to progressive dyspnea and dry cough during the previous 7 days; she had no fever. On physical examination, dry Velcro crackles were audible in both lung bases, with considerable desaturation requiring oxygen supplementation. A high-resolution CT (HRCT) of the chest showed bilateral interstitial infiltrates on the peripheral parenchyma with ground-glass opacities. Furthermore, the hypothesis of pulmonary thrombosis was ruled out and there were a few pulmonary nodules suggesting neoplastic disease; however, there was no lymphangitis. Arterial blood gas analysis revealed severe hypoxemia.

The patient was treated with high-flow oxygen therapy, corticosteroids (methylprednisolone 2 $\mathrm{mg} / \mathrm{kg}$ for 3 days and then prednisone $1 \mathrm{mg} / \mathrm{kg}$ daily), broad-spectrum antibiotic and antifungal therapy, trimethoprim/sulfamethoxazole and oseltamivir, with no clinical improvement, leading to mechanical ventilation. Bronchoalveolar lavage did not show malignant cells or opportunistic infection. Cultures were nonsignificant; cytomegalovirus serologies and blood cultures for aerobic and anaerobic bacteria were negative, as was the test for influenza H1N1 virus. A lung biopsy was performed, revealing anatomopathological findings of diffuse alveolar damage and inflammatory infiltrate consisting mainly of lymphocytes and thickening of interalveolar septa, suggesting idiopathic toxicity associated with drugs: so-called cryptogenic organizing pneumonia (fig. 1 ). No evidence of malignancy was found.

The clinical course was unfavorable, with worsening of respiratory insufficiency leading to the patient's death within 18 days after admission.

Case 2

A 75-year-old male patient presented with dyspeptic symptoms, vomiting and weight loss. No previous history of cigarette smoking was reported. A gastroduodenal endoscopy showed a gastric adenocarcinoma. An additional investigation revealed metastatic nodules in the liver, spleen, pancreas and lung.

In June 2009, he began a chemotherapeutic FOLFOX7m regimen. The patient initially achieved partial response and then stable disease after 9 cycles of treatment, with no major toxicities; the ninth dose was administered in November 2009. Fourteen days after the last cycle, he was admitted with pulmonary insufficiency after a few days of common cold symptoms followed by progressive dyspnea, with no fever. Promptly, he required mechanical ventilation. An HRCT of the chest showed spread bilateral interstitial infiltrates on the peripheral parenchyma and ground-glass opacities (fig. 2), with areas of emphysema. The day after admission, a bilateral pneumothorax led to additional clinical deterioration.

Broad-spectrum antibiotic and antifungal empiric therapy was prescribed, concomitant with highdose corticosteroids. Bronchoalveolar lavage did not show malignant cells or opportunistic infection. Cultures were nonsignificant and the test for influenza H1N1 virus was negative.

The patient died 8 days after admission, before a lung biopsy could be performed.

Case 3

A 64-year-old male patient presented after a left hemicolectomy resection following an intestinal occlusion. The pathological study reported an adenocarcinoma, T3N0M0. His comorbidities included 
diabetes, hypertension, coronaropathy and a history of cigarette smoking, having stopped the habit about 30 years previously.

Adjuvant treatment using the FOLFOX7m regimen began in May 2009 and was completed in October 2009, after 12 cycles had been administered with no major toxicities. In November 2009, physical examination and tomography images showed no evidence of disease. In December 2009, he was hospitalized with progressive dyspnea and dry cough developing in the previous 2 weeks, with fever. Lung auscultation revealed inspiratory crackles in both lung bases, and support with noninvasive ventilation was mandatory.

An HRCT of the chest showed bilateral alveolar infiltrates, ground-glass opacities and thickening of interlobular septa, with a peripheral distribution predominating in the bases ( $\underline{\text { fig. } 3}$ ). The patient was treated with corticosteroids, broad-spectrum antibiotic and antifungal therapy, trimethoprim/sulfamethoxazole and oseltamivir. No clinical improvement was achieved, and refractory hypoxemia and hypercapnia necessitated mechanical ventilation. Similar to the other 2 cases, all the microbiological studies were negative, ruling out an infectious etiology. A lung biopsy was performed, with the following anatomopathological findings: diffuse alveolar damage, inflammatory infiltrate in the proliferative phase and diffuse thickening of interalveolar septa, suggesting idiopathic toxicity associated with drugs. No evidence of malignancy was found.

The worsening of respiratory insufficiency led to the patient's death after 14 days.

\section{Discussion}

Different diagnoses of lung infiltrates in cancer patients following chemotherapy include infections, heart failure, neoplasia, pulmonary bleeding and drug toxicity. Lung toxicity is a very rare complication following treatment with platins. Few incidents of acute lung fibrosis have been reported in patients treated with 5-FU and cisplatin [4].

Since oxaliplatin was marketed and became widely used in clinical practice, not many data suggesting pulmonary toxicity have been documented. Early studies regarding safety found no significant increase in pulmonary complications, except for the dyspnea that may occur in the setting of a hypersensitivity reaction [5]. In 2006, Yagüe et al. [6] first reported a case of interstitial pneumonia induced by oxaliplatin alone.

The manifestations of drug-induced pulmonary injury are usually nonspecific, making an accurate diagnosis difficult. Our 3 patients had a rapidly progressive respiratory insufficiency that was refractory to conventional medical treatment. No infectious causes triggering the clinical picture could be identified. Lymphangitis was also ruled out. Most cases of pulmonary toxicity associated with oxaliplatin reported in the literature had a rapid and untoward course [6-8], similar to what occurred in our patient. It is not known whether prior interstitial lung damage or impairment in respiratory function tests may make the development of oxaliplatin-induced interstitial pneumonitis more likely [8].

There is no objective evidence linking oxaliplatin to direct damage of the lung parenchyma. However, there are data suggesting that oxaliplatin may cause glutathione depletion, which could be involved in the pathogenesis of liver damage caused by the drug [9]. In the lung, glutathione plays a significant role as protector against oxidative damage, and depletion caused by oxaliplatin could be the factor triggering the pulmonary lesions leading to interstitial pneumonitis and subsequent pulmonary fibrosis. 
Muneoka et al. [10] presented the demographic and clinical details of 7 cases of oxaliplatin-related toxicity. The majority of the patients were men, and a median of 8 cycles of the drug were administered before the onset of interstitial pneumonia. However, 2 cases occurred after only 1 or 2 cycles of the regimen. Thus, interstitial pneumonia may occur soon after the initiation of the treatment. It can also occur a few months after the adjuvant treatment, as seen in one of our patients.

As mentioned before, interstitial pneumonia induced by 5-FU appears to be extremely rare [11]. In at least 2 previous reports, interstitial pneumonia did not recur despite the reintroduction of 5-FU/leucovorin alone [10,12]. Once interstitial pneumonia has occurred, cessation of the regimen is mandatory. There is no standard treatment so far. High-dose corticosteroid treatment is commonly given for serious cases of this potentially lethal complication. There are no adequate data suggesting whether treatment with antioxidant agents, such as $\mathrm{N}$-acetylcysteine, may be beneficial.

It should be kept in mind that extended use of some drugs may reveal uncommon but fatal toxicities, and caution should be taken when indicating treatments, particularly in the adjuvant setting.

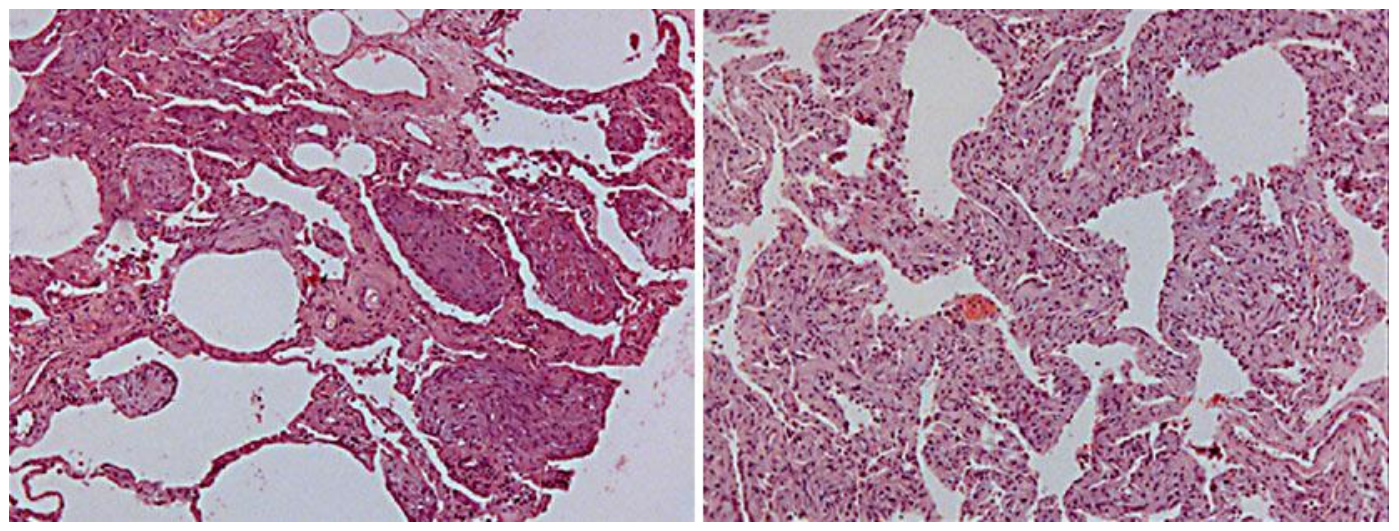

Fig. 1. Diffuse alveolar damage. 

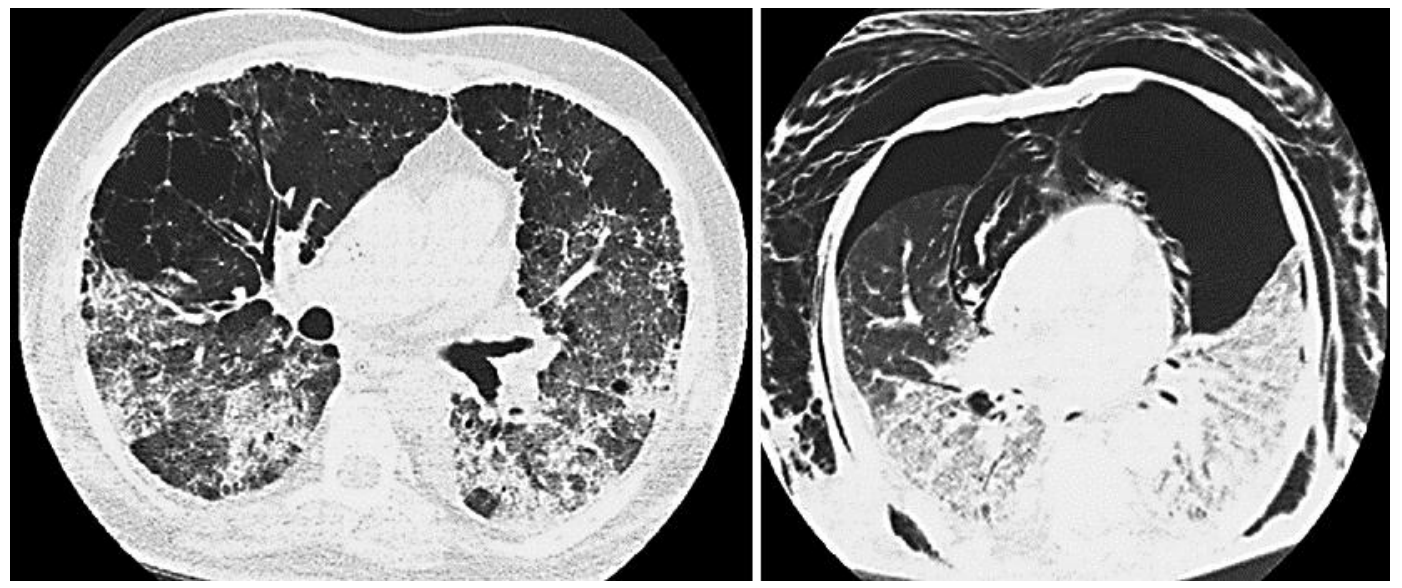

Fig. 2. Bilateral interstitial alveolar infiltrates (left). Bilateral pneumothorax, subcutaneous emphysema and pneumomediastinum (right).
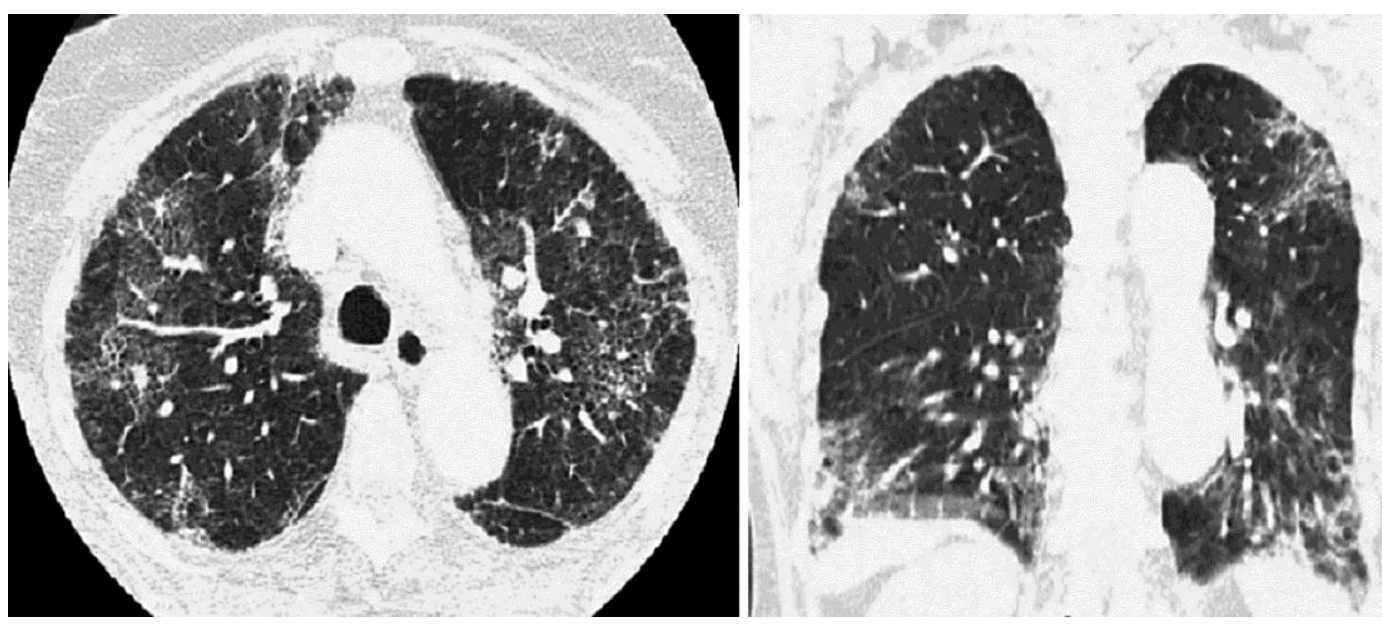

Fig. 3. Interstitial infiltrates on the peripheric parenchyma with ground-glass opacities.

\section{References}

1 Andre T, Boni C, Moudnedji-Boudiaf L, et al: Oxaliplatin, fluorouracil and leucovorin as adjuvant treatment for colon cancer. N Engl J Med 2004;350:2343-2351.

2 Ramanathan RK, Clark JW, Kemeny NE, et al: Safety and toxicity analysis of oxaliplatin combined with fluorouracil or as a single agent in patients with previously treated advanced colorectal cancer. J Clin Oncol 2003;21:2904-2911.

-3 Lobera SA, Marinelarena NS, Echeberría IE, et al: Fatal pneumonitis induced by oxaliplatin. Clin Transl Oncol 2008;10:764-767.

4 Jeanfaivre T, Saint-Andre JP, Tuchais E: Fibrose pulmonaire aigue au decors d'un traitement par fluorouracile et cisplatine. Therapie 1995;50:167-169.

5 Pasetto LM, Monfardini S: Is acute dyspnea related to oxaliplatin administration? World J Gastroenterol 2006;12:5907-5908.

6 Yagüe XH, Soy E, Marino BQ, et al: Interstitial pneumonitis after oxaliplatin treatment in colorectal cancer. Clin Transl Oncol 2005;7:515-517. 
-7 Garrido M, O’Brien A, González S, et al: Cryptogenetic organizing pneumonitis during oxaliplatin chemotherapy for colorectal cancer: case report. Chest 2007;132:1997-1999.

8 Wilcox BE, Ryu JH, Kalra S: Exacerbation of pre-existing interstitial lung disease after oxaliplatin therapy: a report of three cases. Respir Med 2008;102:273-279.

-9 Rubbia-Brandt L, Audard V, Sartoretti P, et al: Severe hepatic sinusoidal obstruction associated with oxaliplatin-based chemotherapy in patients with metastatic colorectal cancer. Ann Oncol 2004;15:460466.

10 Muneoka K, Shirai Y, Sasaki M, et al: Interstitial pneumonia arising in a patient treated with oxaliplatin, 5-fluorouracil, and, leucovorin (FOLFOX). Int J Clin Oncol 2009;14:457-459.

11 Andou H, Itoh K, Tsuda T: A case of fluorouracil-induced pneumonitis. Nihon Kyoubu Shikkan Gakkai Zasshi 1999;35:1080-1083.

12 Gagnadoux F, Roiron C, Carrie E, et al: Eosinophilic lung disease under chemotherapy with oxalip latin for colorectal cancer. Am J Clin Oncol 2002;25:388-390. 